\title{
Kinetic energy contribution to the exchange-correlation energy functional of the extended constrained-search theory
}

\author{
Katsuhiko Higuchi \\ Graduate School of Advanced Sciences of Matter, Hiroshima University, Higashi-Hiroshima 739-8527, Japan \\ Masahiko Higuchi \\ Department of Physics, Faculty of Science, Shinshu University, Matsumoto 390-8621, Japan \\ (Received 30 May 2008; revised manuscript received 5 August 2008; published 13 February 2009)
}

\begin{abstract}
We present the kinetic energy contribution to the exchange-correlation energy functional of the extended constrained-search (ECS) theory by means of the generalized Bauer's relation. Due to the nature of the exchange-correlation energy functional being a function of the Bohr radius and $e^{2}$, three kinds of expressions for the kinetic energy contribution are obtained. These can be utilized as constraints in developing and/or evaluating the approximate form of the exchange-correlation energy functional of the ECS theory. Furthermore, by combining three expressions with the virial relation, we derive other useful relations that include not the kinetic energy contribution but only the exchange-correlation energy functional.
\end{abstract}

DOI: 10.1103/PhysRevA.79.022113

PACS number(s): 71.15.Mb

\section{INTRODUCTION}

In the framework of the density-functional theory (DFT) $[1,2]$, the constrained-search formulation [3-5] was originally proposed by Levy, and was extended to specific cases by several workers. For instance, the electron density and off-diagonal elements of the first-order reduced density matrix were treated as basic variables in Levy's original paper [3]. Also, the extensions of the constrained search formula to the spin-density-functional theory (SDFT) [6], the current-density-functional theory (CDFT) [7], the pair density-functional theory (PDFT) $[8,9]$, and the symmetryadopted version $[10,11]$ have been done so far. Thus, the constrained-search formulation has been pursued in specific cases.

We have previously proposed the generalized framework of the constrained-search formulation, in which arbitrary physical quantities can be chosen as basic variables in addition to the electron density [12-14]. In this framework, the extended version of the constrained-search formula is utilized in defining the universal functional and the kinetic energy functional. Therefore, we call the framework the extended constrained-search (ECS) theory. The validity of the ECS theory has been confirmed by revisiting various kinds of previous theories [12,13] such as the SDFT $[15,16]$, CDFT [17-24], the Hartree-Fock-Kohn-Sham scheme $[25,26]$, and the LDA $+U$ method [27-30]. The ECS theory has the possibility to provide a specially made theory for each individual system, because quantities that characterize the ground state of the system can be chosen as basic variables. Indeed, various kinds of quantities, for example the pair density [31-36], the local density of state at the Fermi level [13], and the spin current density [37] have been chosen as basic variables so far.

In order to perform electronic structure calculations by means of the ECS theory, we have to develop the approximate form of the exchange-correlation energy functional. For this aim, the discussion on how to develop the exchangecorrelation energy functional of the conventional DFT is a good reference. There are, in general, two strategies for developing the approximate form of the exchange-correlation energy functional $[14,38]$. One is to utilize the couplingconstant integration expression for the exchange-correlation energy functional. Various kinds of approximations have been proposed on the basis of the coupling-constant expression in previous theories $[2,17,18,38-44]$. These include the local-density approximation $[2,17,18,38]$, the weighteddensity approximation [38,40-44], and the average-density approximation [38-44]. Along this strategy, we have already derived the coupling-constant integration expression in the ECS theory [14]. This expression is one of the good starting points toward the development of the approximate form.

Another strategy is to employ as constraints exact relations that are fulfilled by the exchange-correlation energy functional. In accordance with this strategy, approximate forms of the exchange and correlation energy functionals have been developed in the previous theories. For example, the generalized gradient approximation (GGA) [45-47], the density-moment expansion method [48-52], the local and variable-separation approximation [53], and the vorticity expansion approximation (VEA) [21-24] have been developed in the DFT, SDFT, and/or CDFT. Concerning the exchangecorrelation energy functional of the ECS theory, some exact relations have already been obtained $[14,54]$. We have presented the virial relation in the ECS theory [14]. Furthermore, by using the coordinate scaling of electrons and adiabatic connection, Nagy has derived exact relations and equations of hierarchy in the ECS theory [54], similarly to the case of the DFT [55,56].

Along the latter strategy, exact relations that are associated with the kinetic energy contribution to the exchangecorrelation energy functional have also been derived in the conventional DFT [26,57-61]. Since it is on the same order of magnitude as the correlation energy for atomic and molecular systems [26,57], the kinetic energy contribution is one of the important issues in the conventional DFT. Bass [58] has derived its useful formula within the conventional DFT by using Bauer's relation [59]. Also, Görling, Levy, and 
Perdew have revisited both Bauer's relation and Bass's expression within the constrained-search formulation of the DFT [61]. An alternative expression for this kinetic energy contribution can be obtained from the virial theorem [60].

In this paper, the kinetic energy contribution to the exchange-correlation energy functional is derived in the framework of the ECS theory by generalizing Bauer's relation. Furthermore, we shall derive the set of exact relations that will be useful in developing and/or evaluating the approximate form of the exchange-correlation energy functional of the ECS theory.

The organization of this paper is as follows. In Sec. II, a generalized version of Bauer's relation is derived in the framework of the ECS theory. In Secs. III and IV, the relation is utilized to present the kinetic energy contribution to the exchange-correlation energy functional of the ECS theory. Three kinds of expressions are presented. Furthermore, some exact relations that can be used as constraints to the exchange-correlation energy functional of the ECS theory are derived in combination with the virial relation. Finally, in Sec. V, we summarize the results and give some comments on them.

\section{GENERALIZATION OF BAUER'S RELATION TO THE ECS THEORY}

With reference to the derivation procedure of Bauer's relation $[59,61]$, let us start with the Hamiltonian that is augmented by an arbitrary operator $\hat{O}$ via a scalar field $\lambda$,

$$
\hat{H}=\hat{T}+g \hat{W}+\int v_{\text {ext }}(\mathbf{r}) \hat{\rho}(\mathbf{r}) \mathrm{d} \mathbf{r}+\lambda \hat{O},
$$

where $\hat{T}, \hat{W}$, and $\hat{\rho}(\mathbf{r})$ are operators of the kinetic energy, electron-electron interaction, and electron density, respectively, and where $g$ denotes the coupling constant for the electron-electron interaction. It should be mentioned that if $\lambda$ and $g$ are equal to 0 and 1 , respectively, then Eq. (1) becomes the actual Hamiltonian of a many-electron system in an external potential $v_{\text {ext }}(\mathbf{r})$.

In the ECS theory [12-14], arbitrary physical quantities can be chosen as basic variables in addition to the electron density $\rho(\mathbf{r})$. Here, suppose that the basic variable chosen is denoted by $\mathbf{X}(\mathbf{r})$. Using the ECS formula, the universal functional is defined as

$$
F[\rho, \mathbf{X}](\lambda, g)=\operatorname{Min}_{\Psi \rightarrow(\rho, \mathbf{X})}\langle\Psi|\hat{T}+g \hat{W}+\lambda \hat{O}| \Psi\rangle,
$$

where $\Psi \rightarrow(\rho, \mathbf{X})$ indicates that the minimization is performed among all antisymmetric wave functions which yield the prescribed $\rho(\mathbf{r})$ and $\mathbf{X}(\mathbf{r})$. Of course, Eq. (2) becomes the usual universal functional $F[\rho, \mathbf{X}]$ in the case of $(\lambda, g)$ $=(0,1)$, i.e., $F[\rho, \mathbf{X}](0,1)=F[\rho, \mathbf{X}]$. It should be noted that since the necessary and sufficient conditions for $N$-representability of $(\rho(\mathbf{r}), \mathbf{X}(\mathbf{r}))$ are unknown except for in specific cases, there may not exist some $\Psi$ that is associated with $(\rho(\mathbf{r}), \mathbf{X}(\mathbf{r}))$ of interest. One possible way to avoid this difficulty was recently proposed by Levy and Ayers [62].

Here we shall give a comment on the existence and uniqueness of the minimum on the right-hand side of Eq. (2).
It is shown that the minimum exists for $v$-representable $(\rho(\mathbf{r}), \mathbf{X}(\mathbf{r}))$, but it is an open question whether the minimum exists or not for $(\rho(\mathbf{r}), \mathbf{X}(\mathbf{r}))$ that is both non- $v$-representable and $N$-representable. Also, whether the minimum is unique or not is the remaining issue that should be discussed. Although such problems are pointed out and discussed in specific cases $[4,5,10,11,15,63-72]$, we shall proceed to a discussion under the assumption that the minimum uniquely exists on the right-hand side of Eq. (2), i.e., $(\rho(\mathbf{r}), \mathbf{X}(\mathbf{r}))$ is supposed to be of that type.

If we assume the differentiability of Eq. (2) and denote the minimizing wave function in Eq. (2) as $\Psi[\rho, \mathbf{X}]_{\lambda g}$, then $\Psi[\rho, \mathbf{X}]_{\lambda g}$ obeys the following equation:

$$
\begin{aligned}
& \left\{\hat{T}+g \hat{W}+\lambda \hat{O}+\int v_{\lambda g}(\mathbf{r}) \hat{\rho}(\mathbf{r}) \mathrm{d} \mathbf{r}+\int \mathbf{a}_{\lambda g}(\mathbf{r}) \cdot \hat{\mathbf{X}}(\mathbf{r}) \mathrm{d} \mathbf{r}\right\} \\
& \quad \times\left|\Psi[\rho, \mathbf{X}]_{\lambda g}\right\rangle=E_{\lambda g}\left|\Psi[\rho, \mathbf{X}]_{\lambda g}\right\rangle,
\end{aligned}
$$

where $E_{\lambda g}, v_{\lambda g}(\mathbf{r})$, and $\mathbf{a}_{\lambda g}(\mathbf{r})$ are the Lagrange multipliers that correspond to constraints on $\Psi[\rho, \mathbf{X}]_{\lambda g}$, i.e., $\Psi[\rho, \mathbf{X}]_{\lambda g}$ is normalized to unity, and yields both $\rho(\mathbf{r})$ and $\mathbf{X}(\mathbf{r})$. From Eq. (3), $E_{\lambda g}$ is given by

$$
\begin{aligned}
E_{\lambda g}= & \left\langle\Psi[\rho, \mathbf{X}]_{\lambda g}\right| \hat{T}+g \hat{W}+\lambda \hat{O}+\int v_{\lambda g}(\mathbf{r}) \hat{\rho}(\mathbf{r}) \mathrm{d} \mathbf{r} \\
& +\int \mathbf{a}_{\lambda g}(\mathbf{r}) \cdot \hat{\mathbf{X}}(\mathbf{r}) \mathrm{d} \mathbf{r}\left|\Psi[\rho, \mathbf{X}]_{\lambda g}\right\rangle .
\end{aligned}
$$

Differentiating both sides of Eq. (4) with respect to $\lambda$ or $g$, we get

$$
\begin{aligned}
\frac{\partial E_{\lambda g}}{\partial \lambda}= & \left\langle\Psi[\rho, \mathbf{X}]_{\lambda g}|\hat{O}| \Psi[\rho, \mathbf{X}]_{\lambda g}\right\rangle+\int \frac{\partial v_{\lambda g}(\mathbf{r})}{\partial \lambda} \rho(\mathbf{r}) \mathrm{d} \mathbf{r} \\
& +\int \frac{\partial \mathbf{a}_{\lambda g}(\mathbf{r})}{\partial \lambda} \cdot \mathbf{X}(\mathbf{r}) \mathrm{d} \mathbf{r}, \\
\frac{\partial E_{\lambda g}}{\partial g}= & \left\langle\Psi[\rho, \mathbf{X}]_{\lambda g}|\hat{W}| \Psi[\rho, \mathbf{X}]_{\lambda g}\right\rangle+\int \frac{\partial v_{\lambda g}(\mathbf{r})}{\partial g} \rho(\mathbf{r}) \mathrm{d} \mathbf{r} \\
& +\int \frac{\partial \mathbf{a}_{\lambda g}(\mathbf{r})}{\partial g} \cdot \mathbf{X}(\mathbf{r}) \mathrm{d} \mathbf{r} .
\end{aligned}
$$

Here, the Hellmann-Feynman theorem has been used. On the other hand, using Eq. (2), we can rewrite Eq. (4) as

$$
E_{\lambda g}=F[\rho, \mathbf{X}](\lambda, g)+\int v_{\lambda g}(\mathbf{r}) \rho(\mathbf{r}) \mathrm{d} \mathbf{r}+\int \mathbf{a}_{\lambda g}(\mathbf{r}) \cdot \mathbf{X}(\mathbf{r}) \mathrm{d} \mathbf{r} .
$$

Differentiating both sides of Eq. (7) with respect to $\lambda$ or $g$, we also get

$$
\begin{aligned}
\frac{\partial E_{\lambda g}}{\partial \lambda}= & \frac{\partial F[\rho, \mathbf{X}](\lambda, g)}{\partial \lambda}+\int \frac{\partial v_{\lambda g}(\mathbf{r})}{\partial \lambda} \rho(\mathbf{r}) \mathrm{d} \mathbf{r} \\
& +\int \frac{\partial \mathbf{a}_{\lambda g}(\mathbf{r})}{\partial \lambda} \cdot \mathbf{X}(\mathbf{r}) \mathrm{d} \mathbf{r},
\end{aligned}
$$




$$
\begin{aligned}
\frac{\partial E_{\lambda g}}{\partial g}= & \frac{\partial F[\rho, \mathbf{X}](\lambda, g)}{\partial g}+\int \frac{\partial v_{\lambda g}(\mathbf{r})}{\partial g} \rho(\mathbf{r}) \mathrm{d} \mathbf{r} \\
& +\int \frac{\partial \mathbf{a}_{\lambda g}(\mathbf{r})}{\partial g} \cdot \mathbf{X}(\mathbf{r}) \mathrm{d} \mathbf{r} .
\end{aligned}
$$

Comparison of Eq. (5) with Eq. (8), and that of Eq. (6) with Eq. (9), leads to

$$
\begin{aligned}
& \frac{\partial F[\rho, \mathbf{X}](\lambda, g)}{\partial \lambda}=\left\langle\Psi[\rho, \mathbf{X}]_{\lambda g}|\hat{O}| \Psi[\rho, \mathbf{X}]_{\lambda g}\right\rangle, \\
& \frac{\partial F[\rho, \mathbf{X}](\lambda, g)}{\partial g}=\left\langle\Psi[\rho, \mathbf{X}]_{\lambda g}|\hat{W}| \Psi[\rho, \mathbf{X}]_{\lambda g}\right\rangle,
\end{aligned}
$$

respectively.

By utilizing Eq. (10), we obtain

$$
\begin{aligned}
& \frac{\partial}{\partial \lambda}\{F[\rho, \mathbf{X}](\lambda, 1)-F[\rho, \mathbf{X}](\lambda, 0)\}_{\lambda=0} \\
& \quad=\left\langle\Psi[\rho, \mathbf{X}]_{01}|\hat{O}| \Psi[\rho, \mathbf{X}]_{01}\right\rangle-\left\langle\Psi[\rho, \mathbf{X}]_{00}|\hat{O}| \Psi[\rho, \mathbf{X}]_{00}\right\rangle
\end{aligned}
$$

Here, we shall suppose that $\rho(\mathbf{r})$ and $\mathbf{X}(\mathbf{r})$ are noninteracting $v$-representable, and that $\mathbf{X}(\mathbf{r})$ is a single-particle property such as the spin density or paramagnetic current density. Under these assumptions, $\Psi[\rho, \mathbf{X}]_{00}$ is equal to the minimizing Slater determinant in the definition of the kinetic energy functional $T_{s}[\rho, \mathbf{X}]=\operatorname{Min}_{\Phi \rightarrow(\rho, \mathbf{X})}\langle\Phi|\hat{T}| \Phi\rangle$, where $\Phi$ denotes the Slater determinant. This is because these assumptions lead to the equation $\operatorname{Min}_{\Psi \rightarrow(\rho, \mathbf{X})}\langle\Psi|\hat{T}| \Psi\rangle$ $=\operatorname{Min}_{\Phi \rightarrow(\rho, \mathbf{X})}\langle\Phi|\hat{T}| \Phi\rangle$, i.e., $F[\rho, \mathbf{X}](0,0)=T_{s}[\rho, \mathbf{X}][14]$. If we denote the minimizing Slater determinant as $\Phi[\rho, \mathbf{X}]$, then we get $\Psi[\rho, \mathbf{X}]_{00}=\Phi[\rho, \mathbf{X}]$. Under this assumption, Eq. (12) is rewritten as

$$
\begin{aligned}
& \frac{\partial}{\partial \lambda}\{F[\rho, \mathbf{X}](\lambda, 1)-F[\rho, \mathbf{X}](\lambda, 0)\}_{\lambda=0} \\
& \quad=\langle\Psi[\rho, \mathbf{X}]|\hat{O}| \Psi[\rho, \mathbf{X}]\rangle-\langle\Phi[\rho, \mathbf{X}]|\hat{O}| \Phi[\rho, \mathbf{X}]\rangle,
\end{aligned}
$$

where we have used the fact that $\Psi[\rho, \mathbf{X}]_{01}$ is identical with the minimizing wave function $\Psi[\rho, \mathbf{X}]$ in the definition of $F[\rho, \mathbf{X}]\left(=\operatorname{Min}_{\Psi \rightarrow(\rho, \mathbf{X})}\langle\Psi|\hat{T}+\hat{W}| \Psi\rangle\right)$. The left-hand side of Eq. (13) can also be obtained by integrating the left-hand side of Eq. (11) with respect to $g$ from 0 to 1 and by differentiating the result with respect to $\lambda$. We have

$$
\begin{aligned}
& \frac{\partial}{\partial \lambda}\{F[\rho, \mathbf{X}](\lambda, 1)-F[\rho, \mathbf{X}](\lambda, 0)\}_{\lambda=0} \\
& \quad=\frac{\partial}{\partial \lambda}\left\{\int_{0}^{1}\left\langle\Psi[\rho, \mathbf{X}]_{\lambda g}|\hat{W}| \Psi[\rho, \mathbf{X}]_{\lambda g}\right\rangle \mathrm{d} g\right\}_{\lambda=0} .
\end{aligned}
$$

Substitution of Eq. (14) into Eq. (13) leads to

$$
\begin{aligned}
& \langle\Psi[\rho, \mathbf{X}]|\hat{O}| \Psi[\rho, \mathbf{X}]\rangle-\langle\Phi[\rho, \mathbf{X}]|\hat{O}| \Phi[\rho, \mathbf{X}]\rangle \\
& \quad=\frac{\partial}{\partial \lambda}\left\{\int_{0}^{1}\left\langle\Psi[\rho, \mathbf{X}]_{\lambda g}|\hat{W}| \Psi[\rho, \mathbf{X}]_{\lambda g}\right\rangle \mathrm{d} g\right\}_{\lambda=0} .
\end{aligned}
$$

Next, we shall associate the right-hand side of Eq. (15) with the exchange-correlation energy functional of the ECS theory. For this purpose, we define the functional $E_{\mathrm{xc}}[\rho, \mathbf{X}](\lambda)$ by

$$
E_{\mathrm{xc}}[\rho, \mathbf{X}](\lambda)=F[\rho, \mathbf{X}](\lambda, 1)-F[\rho, \mathbf{X}](\lambda, 0)-U[\rho],
$$

with

$$
U[\rho]=\frac{e^{2}}{2} \iint \frac{\rho(\mathbf{r}) \rho\left(\mathbf{r}^{\prime}\right)}{\left|\mathbf{r}-\mathbf{r}^{\prime}\right|} \mathrm{d} \mathbf{r} \mathrm{d} \mathbf{r}^{\prime} .
$$

As mentioned above, since $F[\rho, \mathbf{X}](0,1)$ and $F[\rho, \mathbf{X}](0,0)$ are equal to $F[\rho, \mathbf{X}]$ and $T_{s}[\rho, \mathbf{X}]$, respectively, $E_{\mathrm{xc}}[\rho, \mathbf{X}](\lambda)$ becomes the exchange-correlation energy functional $E_{\mathrm{xc}}[\rho, \mathbf{X}]$ of the ECS theory in the case of $\lambda=0$. Namely, we have

$$
E_{\mathrm{xc}}[\rho, \mathbf{X}](0)=E_{\mathrm{xc}}[\rho, \mathbf{X}] .
$$

Using Eq. (11), Eq. (16) is rewritten as

$$
\begin{aligned}
E_{\mathrm{xc}}[\rho, \mathbf{X}](\lambda) & =\int_{0}^{1} \frac{\mathrm{d} F[\rho, \mathbf{X}](\lambda, g)}{\mathrm{d} g} \mathrm{~d} g-U[\rho] \\
& =\int_{0}^{1}\left\langle\Psi[\rho, \mathbf{X}]_{\lambda g}|\hat{W}| \Psi[\rho, \mathbf{X}]_{\lambda g}\right\rangle \mathrm{d} g-U[\rho] .
\end{aligned}
$$

Since $U[\rho]$ is independent of $\lambda$, substitution of Eq. (19) into Eq. (15) leads to

$$
\begin{aligned}
& \langle\Psi[\rho, \mathbf{X}]|\hat{O}| \Psi[\rho, \mathbf{X}]\rangle-\langle\Phi[\rho, \mathbf{X}]|\hat{O}| \Phi[\rho, \mathbf{X}]\rangle \\
& \quad=\left\{\frac{\partial E_{\text {xc }}[\rho, \mathbf{X}](\lambda)}{\partial \lambda}\right\}_{\lambda=0} .
\end{aligned}
$$

If the ground-state values of basic variables are denoted by $\rho_{0}(\mathbf{r})$ and $\mathbf{X}_{0}(\mathbf{r})$, then the ECS theory ensures that $\Psi\left[\rho_{0}, \mathbf{X}_{0}\right]$ is the ground-state wave function $\Psi_{0}$. In addition, $\Phi\left[\rho_{0}, \mathbf{X}_{0}\right]$ is the Slater determinant that is constructed from the solutions for the single-particle equation of the ECS theory. When $\rho(\mathbf{r})=\rho_{0}(\mathbf{r})$ and $\mathbf{X}(\mathbf{r})=\mathbf{X}_{0}(\mathbf{r})$, Eq. (20) becomes

$$
\begin{gathered}
\left\langle\Psi_{0}|\hat{O}| \Psi_{0}\right\rangle-\left\langle\Phi\left[\rho_{0}, \mathbf{X}_{0}\right]|\hat{O}| \Phi\left[\rho_{0}, \mathbf{X}_{0}\right]\right\rangle \\
=\left\{\frac{\partial E_{\mathrm{xc}}\left[\rho_{0}, \mathbf{X}_{0}\right](\lambda)}{\partial \lambda}\right\}_{\lambda=0} .
\end{gathered}
$$

This is the generalization of Bauer's relation. The original Bauer's relation [59] has been successfully used for the derivation of the kinetic energy contribution to the exchange and correlation energy functional of the conventional DFT [58]. In the following section, we shall utilize Eqs. (20) and (21) in order to derive the kinetic energy contribution to the exchange and correlation energy functional of the ECS theory.

\section{KINETIC ENERGY CONTRIBUTION TO $E_{\mathrm{xc}}[\rho, \mathrm{X}]$}

In the ECS theory, the kinetic energy contribution to $E_{\mathrm{xc}}[\rho, \mathbf{X}]$ is expressed as $T[\rho, \mathbf{X}]-T_{s}[\rho, \mathbf{X}][12-14]$, where 
$T[\rho, \mathbf{X}]$ and $T_{s}[\rho, \mathbf{X}]$ are given by $\langle\Psi[\rho, \mathbf{X}]|\hat{T}| \Psi[\rho, \mathbf{X}]\rangle$ and $\langle\Phi[\rho, \mathbf{X}]|\hat{T}| \Phi[\rho, \mathbf{X}]\rangle$, respectively. In order to derive the kinetic energy contribution, let us choose $\hat{T}$ as $\hat{O}$ in Eq. (20). We have

$$
T[\rho, \mathbf{X}]-T_{s}[\rho, \mathbf{X}]=\left\{\frac{\partial E_{\mathrm{xc}}[\rho, \mathbf{X}](\lambda)}{\partial \lambda}\right\}_{\lambda=0} .
$$

This is an expression for the kinetic energy contribution to $E_{\text {xc }}[\rho, \mathbf{X}][73]$. It should be noticed that the right-hand side of Eq. (22) is an incalculable expression. In the DFT, the right-hand side of Eq. (22) is rewritten in a calculable expression by Bass [58]. Namely, it is rewritten in terms of the Bohr radius $\left(a_{B}\right)$ dependence by treating $a_{B}$ as a variable parameter [58]. The calculable expression by Bass is called a useful formula [74], and is applied to the homogeneous electron gas $[58,74]$ and inhomogeneous systems $[58,61]$ in evaluating the kinetic energy contribution to the exchangecorrelation energy functional. Therefore, it is undoubtedly meaningful to replace the incalculable expression [Eq. (22)] with a calculable one. In order to rewrite the right-hand side of Eq. (22) by a calculable expression, we shall formally treat $e^{2}, m$, and $\hbar^{2}$ as variable parameters, although they are fundamental physical constants. As shown below, this enables us to rewrite the right-hand side of Eq. (22) in terms of the $e^{2}, m$, or $\hbar^{2}$ dependence.

In order to get the $\lambda, e^{2}, m$, and $\hbar^{2}$ dependences of $E_{\mathrm{xc}}[\rho, \mathbf{X}](\lambda)$, it is necessary to know those of $\Psi[\rho, \mathbf{X}]_{\lambda g}$. Since $\Psi[\rho, \mathbf{X}]_{\lambda g}$ is the minimizing wave function defined in $\operatorname{Min}_{\Psi \rightarrow(\rho, \mathbf{X})}\langle\Psi|(1+\lambda) \hat{T}+g \hat{W}| \Psi\rangle$, the equation

$$
\begin{aligned}
& \operatorname{Min}_{\Psi \rightarrow(\rho, \mathbf{X})}\langle\Psi|(1+\lambda) \hat{T}+g \hat{W}| \Psi\rangle \\
& \quad=\left\langle\Psi[\rho, \mathbf{X}]_{\lambda g}|(1+\lambda) \hat{T}+g \hat{W}| \Psi[\rho, \mathbf{X}]_{\lambda g}\right\rangle
\end{aligned}
$$

holds. Dividing both sides of this equation by $e^{2}$, we notice that $\Psi[\rho, \mathbf{X}]_{\lambda g}$ is also the minimizing wave function in $\operatorname{Min}_{\Psi \rightarrow(\rho, \mathbf{X})}\left\langle\Psi\left|(1+\lambda)\left(\hbar^{2} / m e^{2}\right) \hat{T}^{\prime}+g \hat{W}^{\prime}\right| \Psi\right\rangle$, where $\hat{T}^{\prime}$ and $\hat{W}^{\prime}$ are defined as $\hat{T}^{\prime}=\left(m / \hbar^{2}\right) \hat{T}$ and $\hat{W}^{\prime}=\left(1 / e^{2}\right) \hat{W}$, respectively. Applying the Lagrange multiplier method, we get the equation for $\Psi[\rho, \mathbf{X}]_{\lambda g}$,

$$
\begin{aligned}
& \left\{(1+\lambda) \frac{\hbar^{2}}{m e^{2}} \hat{T}^{\prime}+g \hat{W}^{\prime}+\int v(\mathbf{r}) \hat{\rho}(\mathbf{r}) \mathrm{d} \mathbf{r}+\int \mathbf{a}(\mathbf{r}) \cdot \hat{\mathbf{X}}(\mathbf{r}) \mathrm{d} \mathbf{r}\right\} \\
& \quad \times\left|\Psi[\rho, \mathbf{X}]_{\lambda g}\right\rangle=\varepsilon\left|\Psi[\rho, \mathbf{X}]_{\lambda g}\right\rangle,
\end{aligned}
$$

where $\varepsilon, v(\mathbf{r})$, and $\mathbf{a}(\mathbf{r})$ stand for the Lagrange multipliers. In order to discuss the $\lambda, e^{2}, m$, and $\hbar^{2}$ dependences of $\Psi[\rho, \mathbf{X}]_{\lambda g}$, we assume that $\hat{\mathbf{X}}(\mathbf{r})$ is normalized by a factor $f\left(e^{2}, m, \hbar^{2}\right)$ so that it is independent of $e^{2}, m$, and $\hbar^{2}$, i.e., $\hat{\mathbf{X}}(\mathbf{r})$ is given by $\hat{\mathbf{x}}(\mathbf{r}) / f\left(e^{2}, m, \hbar^{2}\right)$ if $\hat{\mathbf{x}}(\mathbf{r})$ is the usual physical operator. For example, instead of the paramagnetic current density $\hat{\mathbf{j}}_{p}(\mathbf{r})$, the normalized quantity $(m / \hbar) \hat{\mathbf{j}}_{p}(\mathbf{r})$ is supposed to be chosen as $\hat{\mathbf{X}}(\mathbf{r})$. In Sec. IV, we will discuss this assumption again from a practical point of view.

Under the above-mentioned assumption, we shall first consider the $\lambda, e^{2}, m$, and $\hbar^{2}$ dependences of the Lagrange multipliers $v(\mathbf{r})$ and $\mathbf{a}(\mathbf{r})$. Since these Lagrange multipliers are determined by requiring that $\Psi[\rho, \mathbf{X}]_{\lambda g}$ yields the prescribed basic variables, they can be denoted as $v[\rho, \mathbf{X}](\mathbf{r})$ and $\mathbf{a}[\rho, \mathbf{X}](\mathbf{r})$, respectively. For the present purpose, let us suppose that the Lagrange multipliers are determined for the prescribed $\rho(\mathbf{r})$ and $\mathbf{X}(\mathbf{r})$, and consider the case in which $\lambda$, $e^{2}, m$, and $\hbar^{2}$ are changed such that $(1+\lambda) \hbar^{2} m e^{2}$ is left invariant. In this case, the first and second terms of Eq. (23) are unchanged. If we tentatively substitute $v[\rho, \mathbf{X}](\mathbf{r})$ and $\mathbf{a}[\rho, \mathbf{X}](\mathbf{r})$ into Eq. (23), then the left-hand side of Eq. (23) is exactly the same as before due to the assumption on $\hat{\mathbf{X}}(\mathbf{r})$. Then, we again obtain $\Psi[\rho, \mathbf{X}]_{\lambda g}$ as the solution. Since $\Psi[\rho, \mathbf{X}]_{\lambda g}$ yields the prescribed $\rho(\mathbf{r})$ and $\mathbf{X}(\mathbf{r}), v[\rho, \mathbf{X}](\mathbf{r})$ and $\mathbf{a}[\rho, \mathbf{X}](\mathbf{r})$ are just Lagrange multipliers that are needed in this case. Thus, both $v[\rho, \mathbf{X}](\mathbf{r})$ and $\mathbf{a}[\rho, \mathbf{X}](\mathbf{r})$ are left invariant under the changes such that $(1+\lambda) \hbar^{2} m e^{2}$ is left invariant. Namely, we can say that both $v[\rho, \mathbf{X}](\mathbf{r})$ and $\mathbf{a}[\rho, \mathbf{X}](\mathbf{r})$ depend on $\lambda, e^{2}, m$, and $\hbar^{2}$ only through the factor $(1+\lambda) \hbar^{2} m e^{2}$. This leads to the important fact that $\Psi[\rho, \mathbf{X}]_{\lambda g}$ depends on $\lambda, e^{2}, m$, and $\hbar^{2}$ only through the factor $(1+\lambda) \hbar^{2} m e^{2}$.

In order to express this dependency explicitly, we shall rewrite $\Psi[\rho, \mathbf{X}]_{\lambda g}$ as $\tilde{\Psi}[\rho, \mathbf{X}]\left((1+\lambda)\left(\hbar^{2} / m e^{2}\right), g\right)$. By using this expression, Eq. (19) is rewritten as

$$
\begin{aligned}
\frac{E_{\mathrm{xc}}[\rho, \mathbf{X}](\lambda)}{e^{2}}= & \int_{0}^{1}\left\langle\tilde{\Psi}[\rho, \mathbf{X}]\left((1+\lambda) \frac{\hbar^{2}}{m e^{2}}, g\right)\right| \\
& \times \hat{W}^{\prime}\left|\tilde{\Psi}[\rho, \mathbf{X}]\left((1+\lambda) \frac{\hbar^{2}}{m e^{2}}, g\right)\right\rangle \mathrm{d} g \\
& -\frac{1}{2} \iint \frac{\rho(\mathbf{r}) \rho\left(\mathbf{r}^{\prime}\right)}{\left|\mathbf{r}-\mathbf{r}^{\prime}\right|} \mathrm{d} \mathbf{r} \mathrm{d} \mathbf{r}^{\prime} .
\end{aligned}
$$

The right-hand side of Eq. (24) can be regarded as a function of $(1+\lambda) \hbar^{2} m e^{2}$. If $\widetilde{E}_{\mathrm{xc}}[\rho, \mathbf{X}]\left((1+\lambda)\left(\hbar^{2} / m e^{2}\right)\right)$ is defined as the right-hand side of Eq. (24), then we get

$$
E_{\mathrm{xc}}[\rho, \mathbf{X}](\lambda)=e^{2} \widetilde{E}_{\mathrm{xc}}[\rho, \mathbf{X}]\left((1+\lambda) \frac{\hbar^{2}}{m e^{2}}\right) .
$$

Since $E_{\mathrm{xc}}[\rho, \mathbf{X}](0)$ is equal to $E_{\mathrm{xc}}[\rho, \mathbf{X}]$ as shown in Eq. (18), substitution of $\lambda=0$ into Eq. (25) leads to

$$
E_{\mathrm{xc}}[\rho, \mathbf{X}]=e^{2} \widetilde{E}_{\mathrm{xc}}[\rho, \mathbf{X}]\left(\frac{\hbar^{2}}{m e^{2}}\right) .
$$

This relation means that $E_{\mathrm{xc}}[\rho, \mathbf{X}]$ is a function of the Bohr radius $a_{B}\left(=\hbar^{2} m e^{2}\right)$ and $e^{2}$. Differentiating Eq. (25) with respect to $\lambda$, and changing $e^{2}, m$, and $\hbar^{2}$ as variables, we obtain three kinds of expressions for $\left\{\partial E_{\mathrm{xc}}[\rho, \mathbf{X}](\lambda) / \partial \lambda\right\}_{\lambda=0}$,

$$
\begin{aligned}
\left\{\frac{\partial E_{\mathrm{xc}}[\rho, \mathbf{X}](\lambda)}{\partial \lambda}\right\}_{\lambda=0} & =E_{\mathrm{xc}}[\rho, \mathbf{X}]-e^{2}\left\{\frac{\partial E_{\mathrm{xc}}[\rho, \mathbf{X}]}{\partial\left(e^{2}\right)}\right\}_{\hbar^{2}, m} \\
& =-m\left\{\frac{\partial E_{\mathrm{xc}}[\rho, \mathbf{X}]}{\partial m}\right\}_{\hbar^{2}, e^{2}}
\end{aligned}
$$




$$
=\hbar^{2}\left\{\frac{\partial E_{\mathrm{xc}}[\rho, \mathbf{X}]}{\partial\left(\hbar^{2}\right)}\right\}_{e^{2}, m} .
$$

The reason why three different expressions are obtained is due to the fact that $E_{\mathrm{xc}}[\rho, \mathbf{X}]$ is a function of $a_{B}$ and $e^{2}$ [see Eq. (26)]. By substituting Eqs. (27) into Eq. (22), the kinetic energy contribution to $E_{\mathrm{xc}}[\rho, \mathbf{X}]$ can be obtained as follows:

$$
\begin{aligned}
T[\rho, \mathbf{X}]-T_{s}[\rho, \mathbf{X}] & =E_{\mathrm{xc}}[\rho, \mathbf{X}]-e^{2}\left\{\frac{\partial E_{\mathrm{xc}}[\rho, \mathbf{X}]}{\partial\left(e^{2}\right)}\right\}_{\hbar^{2}, m} \\
& =-m\left\{\frac{\partial E_{\mathrm{xc}}[\rho, \mathbf{X}]}{\partial m}\right\}_{\hbar^{2}, e^{2}} \\
& =\hbar^{2}\left\{\frac{\partial E_{\mathrm{xc}}[\rho, \mathbf{X}]}{\partial\left(\hbar^{2}\right)}\right\}_{e^{2}, m} .
\end{aligned}
$$

The right-hand sides of Eqs. (28) contain the derivatives of $E_{\mathrm{xc}}[\rho, \mathbf{X}]$ that are calculable enough. Thus, the right-hand side of Eq. (22) is rewritten by three kinds of calculable expressions [Eqs. (28)].

Equations (28) show the exact relations between $E_{\mathrm{xc}}[\rho, \mathbf{X}]$ and $T[\rho, \mathbf{X}]-T_{s}[\rho, \mathbf{X}]$. If we get one of the two functionals, then each of Eqs. (28) can be utilized as a constraint to another functional. Also, focusing only on the right-hand sides of Eqs. (28a)-(28c), these equalities can be utilized as constraints in developing the approximate form of $E_{\mathrm{xc}}[\rho, \mathbf{X}]$. It should be noted that the kinetic energy contribution to $E_{\mathrm{xc}}[\rho]$ of the conventional DFT is given in terms of the derivative with respect to not $e^{2}, m$, or $\hbar^{2}$ but the Bohr radius alone [58]. The above-mentioned relations that include $E_{\mathrm{xc}}[\rho, \mathbf{X}]$ alone can be obtained only by expressing the kinetic energy contribution in terms of multiple kinds of derivatives.

The difference $T[\rho, \mathbf{X}]-T_{s}[\rho, \mathbf{X}]$ is alternatively estimated by using the virial relation [14]. The virial relation in the ECS theory has been derived under the assumption that $\mathbf{X}_{0}(\mathbf{r})$ is transformed into $\zeta^{d} \mathbf{X}_{0}(\zeta \mathbf{r})$ by the coordinate scaling of electrons, where $\zeta$ is the scale factor. Under this assumption, the virial relation for isolated systems is given by [14]

$$
\begin{aligned}
& T\left[\rho_{0}, \mathbf{X}_{0}\right]-T_{s}[\rho, \mathbf{X}]+E_{\mathrm{xc}}\left[\rho_{0}, \mathbf{X}_{0}\right] \\
& \quad+\int \rho_{0}(\mathbf{r}) \mathbf{r} \cdot \nabla\left\{\left\{\left.\frac{\delta E_{\mathrm{xc}}[\rho, \mathbf{X}]}{\delta \rho(\mathbf{r})}\right|_{\substack{\rho=\rho_{0} \\
\mathbf{X}=\mathbf{X}_{0}}}\right\} \mathrm{d} \mathbf{r}\right. \\
& \quad+\int \mathbf{X}_{0}(\mathbf{r}) \cdot\left\{\left.(\mathbf{r} \cdot \nabla-d+3) \frac{\delta E_{\mathrm{xc}}[\rho, \mathbf{X}]}{\delta \mathbf{X}(\mathbf{r})}\right|_{\substack{\rho=\rho_{0} \\
\mathbf{X}=\mathbf{X}_{0}}}\right\} \mathrm{d} \mathbf{r}=0 .
\end{aligned}
$$

Combining Eqs. (28) with Eq. (29), we can eliminate $T[\rho, \mathbf{X}]-T_{s}[\rho, \mathbf{X}]$ and obtain the exact relations that include only $E_{\mathrm{xc}}[\rho, \mathbf{X}]$,

$$
\begin{aligned}
- & E_{\mathrm{xc}}\left[\rho_{0}, \mathbf{X}_{0}\right]-\int \rho_{0}(\mathbf{r}) \mathbf{r} \cdot \nabla\left\{\left.\frac{\delta E_{\mathrm{xc}}[\rho, \mathbf{X}]}{\delta \rho(\mathbf{r})}\right|_{\substack{\rho=\rho_{0} \\
\mathbf{X}=\mathbf{X}_{0}}}\right\} \mathrm{d} \mathbf{r} \\
& -\int \mathbf{X}_{0}(\mathbf{r}) \cdot\left\{\left.(\mathbf{r} \cdot \boldsymbol{\nabla}-d+3) \frac{\delta E_{\mathrm{xc} L}[\rho, \mathbf{X}]}{\delta \mathbf{X}(\mathbf{r})}\right|_{\substack{\rho=\rho_{0} \\
\mathbf{X}=\mathbf{X}_{0}}}\right\} \mathrm{d} \mathbf{r} \\
& =E_{\mathrm{xc}}\left[\rho_{0}, \mathbf{X}_{0}\right]-e^{2}\left\{\frac{\partial E_{\mathrm{xc}}\left[\rho_{0}, \mathbf{X}_{0}\right]}{\partial\left(e^{2}\right)}\right\}_{\hbar^{2}, m} \\
& =-m\left\{\frac{\partial E_{\mathrm{xc}}\left[\rho_{0}, \mathbf{X}_{0}\right]}{\partial m}\right\}_{\hbar^{2}, e^{2}} \\
& =\hbar^{2}\left\{\frac{\partial E_{\mathrm{xc}}\left[\rho_{0}, \mathbf{X}_{0}\right]}{\partial\left(\hbar^{2}\right)}\right\}_{e^{2}, m} .
\end{aligned}
$$

Equations (30) can also be utilized as constraints in developing the approximate form of $E_{\mathrm{xc}}[\rho, \mathbf{X}]$. In addition, we may employ Eqs. (30) as sum rules in the so-called virial method $[75,76]$, in which the virial relation (29) is used in calculating the orbital-dependent exchange-correlation potential. It is preferable to use Eqs. (30) in place of Eq. (29), because we do not need $T[\rho, \mathbf{X}]-T_{s}[\rho, \mathbf{X}]$ in Eqs. (30).

Next, we shall divide Eqs. (28) into two parts, i.e., exchange and correlation parts, because the exchange and correlation energy functionals, $E_{x}[\rho, \mathbf{X}]$ and $E_{c}[\rho, \mathbf{X}]$, are often developed in parts as in the case of the GGA [45-47] and VEA [21-24]. Similarly to the DFT, $E_{x}[\rho, \mathbf{X}]$ of the ECS theory is defined by

$$
E_{x}[\rho, \mathbf{X}]=\langle\Phi[\rho, \mathbf{X}]|\hat{W}| \Phi[\rho, \mathbf{X}]\rangle-U[\rho] .
$$

Since it is the minimizing Slater determinant in the definition of $T_{s}[\rho, \mathbf{X}], \Phi[\rho, \mathbf{X}]$ satisfies the equation $\operatorname{Min}_{\Phi \rightarrow(\rho, \mathbf{X})}\left\langle\Phi\left|\hat{T}^{\prime}\right| \Phi\right\rangle=\left\langle\Phi[\rho, \mathbf{X}]\left|\hat{T}^{\prime}\right| \Phi[\rho, \mathbf{X}]\right\rangle$. By applying the Lagrange multiplier method, the equation for $\Phi[\rho, \mathbf{X}]$ can be obtained as follows:

$$
\begin{aligned}
& \left\{\hat{T}+\int v_{s}(\mathbf{r}) \hat{\rho}(\mathbf{r}) \mathrm{d} \mathbf{r}+\int \mathbf{a}_{s}(\mathbf{r}) \cdot \hat{\mathbf{X}}(\mathbf{r}) \mathrm{d} \mathbf{r}\right\}|\Phi[\rho, \mathbf{X}]\rangle \\
& \quad=\varepsilon_{s}|\Phi[\rho, \mathbf{X}]\rangle,
\end{aligned}
$$

where $v_{s}(\mathbf{r}), \mathbf{a}_{s}(\mathbf{r})$, and $\varepsilon_{s}$ are the Lagrange multipliers. In the same way as the discussion below Eq. (23), we can show that both $v_{s}[\rho, \mathbf{X}](\mathbf{r})$ and $\mathbf{a}_{s}[\rho, \mathbf{X}](\mathbf{r})$ are independent of $e^{2}, m$, and $\hbar^{2}$. Therefore, it is concluded that $\Phi[\rho, \mathbf{X}]$ is also independent of them.

Differentiating Eq. (31) with respect to $e^{2}, m$, or $\hbar^{2}$ with the aid of the above-mentioned fact, we get

$$
\begin{gathered}
E_{x}[\rho, \mathbf{X}]-e^{2}\left\{\frac{\partial E_{x}[\rho, \mathbf{X}]}{\partial\left(e^{2}\right)}\right\}_{\hbar^{2}, m}=0, \\
\left\{\frac{\partial E_{x}[\rho, \mathbf{X}]}{\partial m}\right\}_{e^{2}, \hbar^{2}}=0, \\
\left\{\frac{\partial E_{x}[\rho, \mathbf{X}]}{\partial\left(\hbar^{2}\right)}\right\}_{e^{2}, m}=0 .
\end{gathered}
$$


Concerning the correlation energy functional, it is given by $E_{c}[\rho, \mathbf{X}]=E_{\mathrm{xc}}[\rho, \mathbf{X}]-E_{x}[\rho, \mathbf{X}]$. By using Eqs. (33), Eqs. (28) can therefore be rewritten as

$$
\begin{aligned}
T[\rho, \mathbf{X}]-T_{s}[\rho, \mathbf{X}] & =E_{c}[\rho, \mathbf{X}]-e^{2}\left\{\frac{\partial E_{c}[\rho, \mathbf{X}]}{\partial\left(e^{2}\right)}\right\}_{\hbar^{2}, m} \\
& =-m\left\{\frac{\partial E_{c}[\rho, \mathbf{X}]}{\partial m}\right\}_{\hbar^{2}, e^{2}} \\
& =\hbar^{2}\left\{\frac{\partial E_{c}[\rho \mathbf{X}]}{\partial\left(\hbar^{2}\right)}\right\}_{e^{2}, m} .
\end{aligned}
$$

These exact relations Eqs. (33) and (34) can be employed as constraints in devising the approximate form of $E_{x}[\rho, \mathbf{X}]$ and $E_{c}[\rho, \mathbf{X}]$ as well as in the virial method $[75,76]$.

\section{DISCUSSION}

In this section, we shall discuss the assumption that $\hat{\mathbf{X}}(\mathbf{r})$ is independent of $e^{2}, m$, and $\hbar^{2}$. Let us consider the case in which $\hat{\mathbf{x}}(\mathbf{r})$ is chosen as the basic variable instead of $\hat{\mathbf{X}}(\mathbf{r})$. Here recall that $\hat{\mathbf{x}}(\mathbf{r})$ is the operator of the usual physical quantity and is related to $\hat{\mathbf{X}}(\mathbf{r})$ as $\hat{\mathbf{X}}(\mathbf{r})=\hat{\mathbf{x}}(\mathbf{r}) / f\left(e^{2}, m, \hbar^{2}\right)$. The universal functional of this case is defined by $f[\rho, \mathbf{x}]$ $=\operatorname{Min}_{\Psi \rightarrow(\rho, \mathbf{x})}\langle\Psi|\hat{T}+\hat{W}| \Psi\rangle$. The search area in this definition is the set of antisymmetric wave functions that yield both $\rho(\mathbf{r})$ and $\mathbf{x}(\mathbf{r})$. It should be noticed that antisymmetric wave functions in this set simultaneously yield both $\rho(\mathbf{r})$ and $\mathbf{X}(\mathbf{r})$. Therefore, the set of antisymmetric wave functions yielding both $\rho(\mathbf{r})$ and $\mathbf{x}(\mathbf{r})$ coincide with that of antisymmetric wave functions yielding both $\rho(\mathbf{r})$ and $\mathbf{X}(\mathbf{r})$. Consequently, it can be concluded that $\operatorname{Min}_{\Psi \rightarrow(\rho, \mathbf{x})}\langle\Psi|\hat{T}+\hat{W}| \Psi\rangle=\operatorname{Min}_{\Psi \rightarrow(\rho, \mathbf{X})}\langle\Psi| \hat{T}$ $+\hat{W}|\Psi\rangle$. This equation leads to

$$
\Psi[\rho, \mathbf{x}]=\Psi[\rho, \mathbf{X}],
$$

where $\Psi[\rho, \mathbf{x}]$ is the minimizing wave function in the definition of $f[\rho, \mathbf{x}]$. The same discussion holds for the minimizing Slater determinant in the kinetic energy functional. Namely, we have

$$
\Phi[\rho, \mathbf{x}]=\Phi[\rho, \mathbf{X}],
$$

where $\Phi[\rho, \mathbf{x}]$ is the minimizing Slater determinant in $\operatorname{Min}_{\Phi \rightarrow(\rho, \mathbf{x})}\langle\Phi|\hat{T}| \Phi\rangle\left(=t_{s}[\rho, \mathbf{x}]\right)$. Using Eqs. (35) and (36), we can conclude the following equations:

$$
\begin{gathered}
\varepsilon_{\mathrm{xc}}[\rho, \mathbf{x}]=E_{\mathrm{xc}}[\rho, \mathbf{X}], \\
\varepsilon_{x}[\rho, \mathbf{x}]=E_{x}[\rho, \mathbf{X}], \\
\varepsilon_{c}[\rho, \mathbf{x}]=E_{c}[\rho, \mathbf{X}],
\end{gathered}
$$

where $\varepsilon_{\mathrm{xc}}[\rho, \mathbf{x}]$ is the exchange-correlation energy functional of the ECS theory including $\mathbf{x}(\mathbf{r})$ as a basic variable.
On the other hand, by means of the relation $\mathbf{x}(\mathbf{r})$ $=f\left(e^{2}, m, \hbar^{2}\right) \mathbf{X}(\mathbf{r})$, the functional of $\rho(\mathbf{r})$ and $\mathbf{X}(\mathbf{r})$ is formally obtained from that of $\rho(\mathbf{r})$ and $\mathbf{x}(\mathbf{r})$. For example, we have

$$
\varepsilon_{\mathrm{xc}}[\rho, \mathbf{x}]=\varepsilon_{\mathrm{xc}}\left[\rho, f\left(e^{2}, m, \hbar^{2}\right) \mathbf{X}\right]=: \bar{\varepsilon}_{\mathrm{xc}}[\rho, \mathbf{X}],
$$

where $\bar{\varepsilon}_{\mathrm{xc}}[\rho, \mathbf{X}]$ is the functional of $\rho(\mathbf{r})$ and $\mathbf{X}(\mathbf{r})$. From Eqs. (37) and (40), we can immediately construct $\bar{\varepsilon}_{\mathrm{xc}}[\rho, \mathbf{X}]$ $\left(=E_{\mathrm{xc}}[\rho, \mathbf{X}]\right)$ and evaluate it by means of exact relations that are derived in the previous section.

These discussions give the conditions that indicate which type of quantities can be included in the formula of the kinetic energy contribution. For example, if we choose the paramagnetic current density as $\mathbf{X}(\mathbf{r})$, then the present results are applicable as mentioned above. However, that is not the case if the sum of the paramagnetic current density and spincurrent density is chosen as $\mathbf{X}(\mathbf{r})$. Thus, the assumption introduced is not a restriction in usual cases.

\section{CONCLUDING REMARKS}

In the framework of the ECS theory, we have derived the generalized version of Bauer's relation [59]. The relation represents the difference between expectation values of an arbitrary quantity with respect to the ground-state wave function and that with respect to the Kohn-Sham determinant of the ECS theory. This expression is similar to that of the conventional DFT, except that the arbitrary physical quantity $\mathbf{X}(\mathbf{r})$ is added to it as the basic variable.

The generalized Bauer's relation is successfully used to derive the kinetic energy contribution to the exchangecorrelation energy functional. Since the exchange-correlation energy functional of the ECS theory can be expressed as a function of the Bohr radius and $e^{2}$, three kinds of expressions for the kinetic energy contribution are derived. That is, the kinetic energy contribution is expressed in terms of three kinds of derivatives with respect to $e^{2}, m$, or $\hbar^{2}$, while it is in terms of the derivative with respect to the Bohr radius alone in Bass's expression of the conventional DFT [58].

Here, we shall give a comment on the importance of this type of work. As mentioned in Sec. I, exact relations have been effectively used in developing the approximate form of the exchange-correlation energy functional in the DFT, SDFT, CDFT, and so on. For example, the GGA is also developed with the aid of many kinds of exact relations. It is one of the established methods to utilize as constraints exact relations that are fulfilled by the exchange-correlation energy functional. Many works concerning exact relations have been done so far. We derive exact relations in this paper. Their exact relations will be utilized as constraints in developing and/or evaluating the approximate form of the exchange-correlation energy functional of the ECS theory.

Furthermore, by means of these expressions, we can eliminate the kinetic energy contribution term in the virial relation of the ECS theory. The resultant relations [Eqs. (30)] include not the kinetic energy contribution but the exchangecorrelation energy functional alone, which also could be strong sum rules not only for the ECS theory [12-14] but also for the virial method $[75,76]$. Let us go into details. In 
the virial method, the virial relation [Eq. (29)] is utilized in calculating the exchange-correlation potential. Since the virial relation includes the kinetic energy contribution, we need it in order to obtain the exchange-correlation potential. As mentioned in the paper, we can eliminate the kinetic energy contribution term in the virial relation by using exact relations obtained here. Consequently, we may calculate the exchange-correlation potential without the kinetic energy contribution. This is an example of an effective application of exact relations obtained in this paper.

\section{ACKNOWLEDGMENTS}

This work was partially supported by Grant-in-Aid for Scientific Research (No. 19540399) and for Scientific Research in Priority Areas (No. 17064006) of the Ministry of Education, Culture, Sports, Science, and Technology, Japan.
[1] P. Hohenberg and W. Kohn, Phys. Rev. 136, B864 (1964).

[2] W. Kohn and L. J. Sham, Phys. Rev. 140, A1133 (1965).

[3] M. Levy, Proc. Natl. Acad. Sci. U.S.A. 76, 6062 (1979).

[4] M. Levy, Phys. Rev. A 26, 1200 (1982).

[5] E. H. Lieb, Int. J. Quantum Chem. 24, 243 (1983); in Density Functional Methods in Physics, Vol. 123 of NATO Advanced Study Institute Series B: Physics, edited by R. M. Dreizler and J. da Providencia (Plenum, New York, 1985), p. 31. See also Physics as Natural Philosophy, edited by A. Shimony and H. Freshbach (MIT Press, Cambridge, 1982), p. 111.

[6] J. P. Perdew and A. Zunger, Phys. Rev. B 23, 5048 (1981).

[7] S. Erhard and E. K. U. Gross, Phys. Rev. A 53, R5 (1996).

[8] P. Ziesche, Phys. Lett. A 195, 213 (1994).

[9] Á. Nagy, Phys. Rev. A 66, 022505 (2002).

[10] A. Görling, Phys. Rev. A 59, 3359 (1999).

[11] A. Görling, Phys. Rev. Lett. 85, 4229 (2000).

[12] M. Higuchi and K. Higuchi, Phys. Rev. B 69, 035113 (2004).

[13] K. Higuchi and M. Higuchi, Phys. Rev. B 69, 165118 (2004).

[14] K. Higuchi and M. Higuchi, Phys. Rev. B 71, 035116 (2005).

[15] U. von Barth and L. Hedin, J. Phys. C 5, 1629 (1972).

[16] M. M. Pant and A. K. Rajagopal, Solid State Commun. 10, 1157 (1972).

[17] G. Vignale and M. Rasolt, Phys. Rev. Lett. 59, 2360 (1987).

[18] G. Vignale and M. Rasolt, Phys. Rev. B 37, 10685 (1988).

[19] M. Higuchi and A. Hasegawa, J. Phys. Soc. Jpn. 66, 149 (1997).

[20] M. Higuchi and A. Hasegawa, J. Phys. Soc. Jpn. 67, 2037 (1998).

[21] K. Higuchi and M. Higuchi, Phys. Rev. B 74, 195122 (2006).

[22] K. Higuchi and M. Higuchi, Phys. Rev. B 75, 159902(E) (2007).

[23] M. Higuchi and K. Higuchi, Phys. Rev. B 75, 195114 (2007).

[24] K. Higuchi and M. Higuchi, J. Phys.: Condens. Matter 19, 365216 (2007).

[25] S. Baroni and E. Tuncel, J. Chem. Phys. 79, 6140 (1983).

[26] R. G. Parr and W. Yang, Density-Functional Theory of Atoms and Molecules (Oxford University Press, New York, 1989), and references therein.

[27] A. I. Liechtenstein, V. I. Anisimov, and J. Zaanen, Phys. Rev. B 52, R5467 (1995)

[28] V. I. Anisimov, J. Zaanen, and O. K. Andersen, Phys. Rev. B 44, 943 (1991).

[29] V. I. Anisimov and O. Gunnarsson, Phys. Rev. B 43, 7570 (1991).

[30] V. I. Anisimov, I. V. Solovyev, M. A. Korotin, M. T. Czyzyk, and G. A. Sawatzky, Phys. Rev. B 48, 16929 (1993).

[31] M. Higuchi and K. Higuchi, Physica B 387, 117 (2007).
[32] M. Higuchi and K. Higuchi, J. Magn. Magn. Mater. 310, 990 (2007).

[33] M. Higuchi and K. Higuchi, Phys. Rev. A 75, 042510 (2007).

[34] M. Higuchi and K. Higuchi, Phys. Rev. B 78, 125101 (2008).

[35] K. Higuchi and M. Higuchi, J. Phys.: Condens. Matter 21, 064206 (2009).

[36] K. Higuchi and M. Higuchi (unpublished).

[37] S. Rohra and A. Görling, Phys. Rev. Lett. 97, 013005 (2006).

[38] M. Higuchi and K. Higuchi, Phys. Rev. B 65, 195122 (2002).

[39] O. Gunnarsson and P. Johansson, Int. J. Quantum Chem. 10, 307 (1976).

[40] O. Gunnarsson, M. Jonson, and B. I. Lundqvist, Solid State Commun. 24, 765 (1977).

[41] O. Gunnarsson, M. Jonson, and B. I. Lundqvist, Phys. Rev. B 20, 3136 (1979).

[42] O. Gunnarson, and R. O. Jones, Phys. Scr. 21, 394 (1980).

[43] J. A. Alonso and L. A. Girifalco, Solid State Commun. 24, 135 (1977).

[44] J. A. Alonso and L. A. Girifalco, Phys. Rev. B 17, 3735 (1978).

[45] J. P. Perdew and Y. Wang, Phys. Rev. B 33, 8800 (1986).

[46] J. P. Perdew, in Electronic Structure of Solids '91, edited by P. Ziesche and H. Eschrig (Akademie Verlag, Berlin, 1991), p. 11.

[47] J. P. Perdew, K. Burke, and M. Ernzerhof, Phys. Rev. Lett. 77, 3865 (1996).

[48] S. Liu, Á. Nagy, and R. G. Parr, Phys. Rev. A 59, 1131 (1999).

[49] Á. Nagy, S. B. Liu, and R. G. Parr, Phys. Rev. A 59, 3349 (1999).

[50] E. Bene and Á. Nagy, Chem. Phys. Lett. 324, 475 (2000).

[51] S. Liu, F. De Proft, A. Nagy, and R. G. Parr, Adv. Quantum Chem. 36, 77 (1999).

[52] P. W. Ayers, J. B. Lucks, and R. G. Parr, Acta Phys. Chim. Debrecina 34-35, 223 (2002).

[53] S. Liu, Phys. Rev. A 54, 1328 (1996).

[54] Á. Nagy, Int. J. Quantum Chem. 106, 1043 (2006).

[55] Á. Nagy, Phys. Rev. A 47, 2715 (1993).

[56] S. B. Liu and R. G. Parr, Phys. Rev. A 53, 2211 (1996).

[57] C. O. Almbladh and A. C. Pedroza, Phys. Rev. A 29, 2322 (1984).

[58] R. Bass, Phys. Rev. B 32, 2670 (1985).

[59] G. E. W. Bauer, Phys. Rev. B 27, 5912 (1983).

[60] M. Levy and J. P. Perdew, Phys. Rev. A 32, 2010 (1985).

[61] A. Görling, M. Levy, and J. P. Perdew, Phys. Rev. B 47, 1167 (1993).

[62] P. W. Ayers and M. Levy, J. Chem. Sci. 117, 507 (2005).

[63] H. Englisch and R. Englisch, Physica A 121, 253 (1983). 
[64] K. Capelle and G. Vignale, Phys. Rev. Lett. 86, 5546 (2001).

[65] K. Capelle and G. Vignale, Phys. Rev. B 65, 113106 (2002).

[66] K. Capelle and V. L. Libero, Int. J. Quantum Chem. 105, 679 (2005).

[67] W. Kohn, A. Savin, and C. A. Ullrich, Int. J. Quantum Chem. 100, 20 (2004)

[68] C. A. Ullrich, Phys. Rev. B 72, 073102 (2005).

[69] P. W. Ayers and W. T. Yang, J. Chem. Phys. 124, 224108 (2006).

[70] T. Heaton-Burgess, P. Ayers, and W. T. Yang, Phys. Rev. Lett. 98, 036403 (2007).

[71] H. Eschrig and W. E. Pickett, Solid State Commun. 118, 123 (2001).
[72] S. Pittalis, S. Kurth, N. Helbig, and E. K. U. Gross, Phys. Rev. A 74, 062511 (2006).

[73] A different type of expression has been derived by Nagy [54]. Whereas in the expression by Nagy $\lambda$ denotes both the scale factor of electron coordinates and the coupling constant of electron-electron interaction, in Eq. (22) $\lambda$ stands for a scalar field that is coupled with $\hat{O}$.

[74] R. M. Dreizler and E. K. U. Gross, Density Functional Theory (Springer-Verlag, Berlin, 1990).

[75] M. Kodera, K. Higuchi, A. Narita, and M. Higuchi, J. Phys. Soc. Jpn. 76, 054302 (2007).

[76] M. Kodera, K. Higuchi, A. Narita, and M. Higuchi, Phys. Rev. A 78, 012501 (2008) 